\title{
A estabilidade das resinas tipo bulk fill na dentística reabilitadora
}

\author{
The stability of bulk fill resins in the rehabilitating dentist
}

La estabilidad de las resinas a granel en la odontología rehabilitante

José Milton de Aquino e Silva Neto ${ }^{1 *}$, Mariana Freitas Ferreira ${ }^{1}$, Yara da Silva Barros ${ }^{1}$, Sysllânia Vitória Pôrto Souza ${ }^{1}$, João Vitor Brasil Alves Rodrigues Almeida Barros ${ }^{1}$, Júllian Karen Bezerra dos Santos ${ }^{1}$, Michelle Leão Bittencourt Brandão Medeiros ${ }^{1}$, Tayguara Cerqueira Cavalcanti'.

\section{RESUMO}

Objetivo: Avaliar e contextualizar a estabilidade das Resinas Compostas do tipo bulk fill (RCBF) analisando os aspectos mecânicos, suas possíveis vantagens e desvantagens ao que se refere à resistências e contrações. Métodos: Tratou-se de uma revisão de literatura, com abordagem descritiva e caráter informativo, onde o processo de formulação se deu através de buscas por literaturas cientificas, em bases de dados, tendo como descritores: A importância da resina composta do tipo Bulk fill, sua estabilidade, utilização da polimerização e potência de irradiação. Resultados: Ao que se refere a RCBF pode ser constatado boa estabilidade, apresentando material avançado não só no aspecto estético como também em sua forma físicas e mecânicas, esse fato se deve por causa da sua matriz apresentar monômeros com propriedades avançadas dos quais diminuem de forma significativa a tensão de polimerização, tornando os monômeros mais ajustáveis permitindo assim maior ligação e adição de fotoiniciadores que sendo relacionados à canforoquinona aperfeiçoa o nível de conversão das moléculas. Considerações Finais: As RCBF são sugeridas para a restauração dos elementos dentais posteriores dos quais apontam certa estabilidade de cor, força química e mecânica, porém é necessário estudos longitudinais para analisar a estabilidade de suas propriedades a longo prazo.

Palavras-chave: Reações químicas, Estética, Polimerização, Força compressiva.

\begin{abstract}
Objective: Evaluate and contextualize the stability of Bulk Fill Composite Resins (RCBF) by analyzing the mechanical aspects, their possible advantages and disadvantagens with regard to resistance and contractions. Methods: It was a literature review, with a descriptive and informative approach, where the formulation process took place through searches for scientific literature, in databases, having as descriptors: The importance of Bulk fill composite resin, its stability, use of polymerization and irradiation power. Results: With regard to RCBF, good stability can be seen, presenting advanced material not only in the aesthetic aspect but also in its physical and mechanical form, this fact is prevented by the fact that its matrix presentes monomers with advanced properties, which significantly decrease the polymerization tension, making the monomers more adjustable, thus allowing greater binding and addition of photoinitiators which, being related to camphorquinone, improves the level of conversion of the molecules. Final Considerations: RCBF are suggested for the restoration of posterior dental elements, which indicate a certain color stability, chemical and mechanical strength, however longitudinal studies are necessary to analyze the stability of their properties in the long term.
\end{abstract}

Keywords: Chemical reactions, Esthetics, Polymerization, Compressive strength.

${ }^{1}$ Centro Universitário CESMAC (CESMAC), Maceió - AL.

*E-mail: Milton_neto_166@hotmail.com

SUBMETIDO EM: 5/2020

ACEITO EM: 6/2020

PUBLICADO EM: 8/2020

REAS/EJCH | Vol.Sup.n.58 | e4085 | DOI: https://doi.org/10.25248/reas.e4085.2020 Página 1 de $\mathbf{1 2}$ 


\section{RESUMEN}

Objetivo: Evaluar y contextualizar la estabilidad de las resinas compuestas de relleno a granel (RCBF) mediante el análisis de los aspectos mecânicos, sus posibles ventajas y desventajas con respecto a la resistencia y las contracciones. Métodos: Fue una revisión de literatura, con un enfoque descriptivo e informativo, donde el proceso de formulación se llevó a cabo mediante búsquedas de literatura científica, en bases de datos, teniendo como descriptores: La importancia de la resina compuesta de relleno a granel, su estabilidade, uso de polimerización y poder de irradiación. Resultados: Con respecto al RCBF, se puede ver una buena estabilidad, presentando material avanzado no solo en el aspecto estético sino también en su forma física y mecánica, este hecho se evita por el hecho de que su matriz presenta monómeros con propiedades avanzadas, lo que disminuye significativamente tensión de polimerización, lo que hace de los monómeros sean más ajustables, lo que permite una mayor unión y adición de fotoiniciadores que, al estar relacionados con la alcanforquinona, mejora el nivel de conversión de las moléculas. Consideraciones finales: Se recomiendan RCBF para la restauración de elementos dentales posteriores, que indican una cierta estabilidad del color, resistencia química y mecánica, sin embargo, se necesitan estúdios longitudinales para analizar la propiedades a largo plazo.

Palabras Clave: Reacciones químicas, Estética, Polimerizacion, Fuerza compresiva.

\section{INTRODUÇÃO}

A elevada busca por aparatos que apresentem enormes características ao que se refere à qualidade dos materiais restauradores nos elementos dentários e que se encontrem semelhantes com as expostas pelas substâncias que estão presentes na dentição é bastante alta nos dias que correm, sendo tornados sempre em consideração no momento da sua seleção: Entre eles são visto os fatores relacionados a aplicabilidade, físicos, funcionalidades e estéticos (RODRIGUES JUNIOR e COSTA E, 2016).

Ao ser observado tais necessidades e exigências para o processo restaurador dos elementos dentários, surgiu assim os materiais restauradores, podendo ser destacado as resinas compostas (RC), dos quais começaram a ser viabilizados, levando em consideração os aspectos principais e fundamentais, que são: a anatomia dentaria, aspectos estéticos e sua funcionalidade mecânica, de forma aceitável. Desse modo, o processo evolutivo dos componentes resinosos foi além do esperado no que se refere ao princípio básico de adesividade e coesividade na restauração dentária. Modificações realizadas nos princípios constituintes desses respectivos materiais, por exemplo nas modificações estabelecidas nos tamanhos, na acomodação dos fragmentos presentes nas partículas e nos seus pesos moleculares, disponibilizaram melhoramentos apreciáveis em seus atributos. Com isso teve como finalidade a produção de materiais com melhor qualidade e durabilidade ao polimento e mais resistente a degradação (FERRACANE JL, 2011).

Porém mesmo sabendo desses fatores que levam a determinadas limitações Anusavice KJ (2005), ressalta que mesmo as resinas possuindo determinados fatores limitantes elas possuem agentes poliméricos, dos quais em sua profundidade são apinhados de ligações cruzadas e reestruturada por um espalhamento de vidros e cristais vigentes, além dos fragmentos presentes nas cargas orgânicas. Em outro estudo foi analisado que com o passar do tempo as RC são utilizadas também nos elementos dentais posteriores, podendo ser feito modificações dos elementos dentais que possuam amálgama e até mesmo em eventuais restaurações de prótese indiretas.

Porém deve ser levado em consideração que um dos motivos que fazem com quer haja a constituição do bom resultado dos quais é composto a durabilidade do procedimento realizado, é estabelecido sobretudo da competência e capacidade técnica do profissional, o Cirurgião dentista deve deixar o paciente ciente ao que se refere ao processo de instrução e motivação oral (KAYA MS, et al., 2018).

Com a finalidade de confirmar a transformação certa no que se refere a quantidade de RC aplicada na cavidade dental e minimizar as defluências na contração, verificou-se o estabelecimento de procedimentos incrementais, dos quais foram visualizados seguimentos nos tratamentos mais aceitáveis e satisfatórios, em 
que foi estabelecido os ajustamentos onde cada incrementação de resina composta tem que dispor no valor extremado de $2 \mathrm{~mm}$ de espessidão (FLURY S, et al., 2014). No entanto, foi constatado que essa forma metodológica empregada, quando são colocados pelo Cirurgião Dentista (CD) em cavidades extensas ou que apresentem uma maior dificuldade de acesso exigem maior quantidade de tempo, além de que aumenta possíveis riscos de contaminações cruzada (SILVA NETO JM, et al., 2019).

Buscando o melhor atendimento e tempo de procedimento de um paciente para outro, sem esquecer a qualidade do serviço prestado, foram criadas as resinas compostas do tipo bulk fill, onde exige apenas aplicação única do profissional. Os cientistas que desenvolveram essa resina ressaltaram que esse tipo específico é bastante indicada, principalmente quando os preparos cavitários apresentam profundidades maiores que $4 \mathrm{~mm}$, uma vez que, em contraposição as resinas convencionais, apresentam modificações se forem aplicadas de uma única vez, principalmente no que se refere as modificações presentes nos compostos e porção monomérica, na adição da nitidez, clareza e na abrandamento do composto inorgânico presentes (SILVA NETO JM, et al.,2019).

Fundamentando esses fatores, o estudo tem como foco principal fazer uma revisão de literatura sobre a implantação da Resinas Compostas do tipo bulk fill (RCBF) nos consultórios odontológicos, averiguando seus componentes e sua estabilidade de cor, força química e mecânica, visto que há uma escassez ao que se refere a esses aspectos, com o intuito de analisar se elas possuem melhores resultados quando comparadas as convencionais.

\section{MÉTODOS}

Para a elaboração deste trabalho foi executada uma revisão de literatura, sendo realizada entre o período de dezembro de 2019 a abril de 2020, dos quais foram examinados artigos divulgados em bases de dados eletrônicos LILACS (Literatura Latino Americana e do Caribe em Ciências da Saúde), MEDLINE (Literatura Internacional em Ciência da Saúde) e SCIELO (Scientific Electronic Library Online) e trabalhos científicos que estavam disponíveis nos acervos bibliográficos presentes na biblioteca central do Centro Universitário CESMAC (Centro de Ensino Superior de Maceió).

Foram consultados textos em língua portuguesa e inglesa, em que referira-se a estabilidade das cores das resinas Compostas do tipo bulk fill (RCBF) após sua aplicação, sua força química e mecânica e o tempo de longevidade, possuindo como forma de restrições os períodos aos quais eles foram publicados, os artigos que não estavam presentes na integra, relatos de casos, séries de casos, ensaios não controlados e os artigos que não dispunham expressividade com a temática estabelecida, sendo eleitos os textos científicos que exibiam e constavam o real objetivo do trabalho, analisando a relevância da utilização das Resinas Compostas do tipo bulk fill (RCBF) na área odontológica certificando o melhor procedimento para a aquisição de seguimentos aceitáveis e satisfatórios para o paciente, sendo selecionadas publicações científicas no período entre 2010 e 2020, com ênfase no período entre 2014 e 2019, dispondo dos seguintes descritores: resina composta, durabilidade, tempo de polimerização e estabilidade ao termino do tratamento.

\section{RESULTADOS E DISCUSSÃO}

Ao iniciar a pesquisa, foram adquiridos 26 artigos relativos à utilização das Resinas Compostas do tipo bulk fill (RCBF) referente a sua estabilidade. Cujo, 13 apresentavam repetições nas referidas bases de dados, ficando assim 13 artigos para o processo de leitura, dos quais 3 foram eliminados por não corresponderem as principais adaptações dos critérios que estavam sendo estabelecidos. 10 artigos eleitos para análise dos resumos, com isso um foi eliminado por não apresentar objetividade com a temática, ficando apenas 9 para certificar esse estudo. Após a apreciação das referências dos artigos nenhum outro trabalho foi acrescentado. Ao fim das averiguações dos respectivos dados, a revisão foi mesclada remediando o processo de miscigenação dos nove artigos, com isso, o fluxograma da Figura 1 demostra de forma transparente todas as metodologias de busca pelas pesquisas elegidas para a confecção da respectiva revisão. 
Figura 1 - Fluxograma dos estudos identificados.

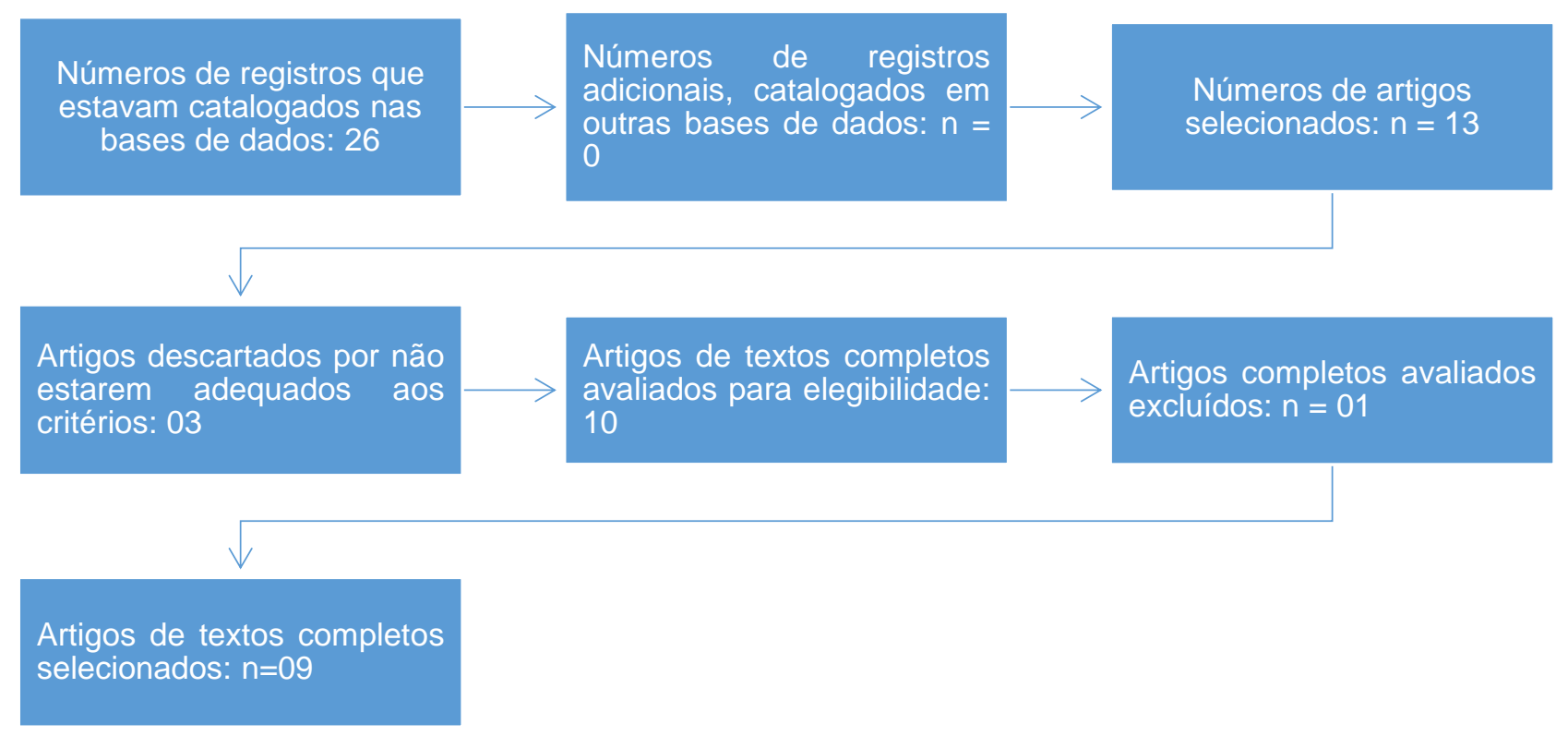

Fonte: Neto JMAS, et al., 2020.

O modo pelos quais as resinas bulk-fill mantêm-se em processo de constrição de suas capacidades, provavelmente diminuída, é muito alterável, do qual cada fabricante expõe suas particularidades tecnologia. Dos conceitos estabelecidos e manipulados pelos inúmeros fabricantes, pode-se indicar a aplicação dos monômeros característicos, monômeros coadjuvantes, dessemelhantes fotoiniciadores, incorporação de distintas cargas inorgânicas como as fibras de vidro e aplicação da força energética ultrassônica precedentemente à sua fotopolimerização. A explicação ao que se refere as concepções de concentrações, mecanismos e sua forma de polimerização estão expostos no Quadro 2. 
Quadro 1 - Dados obtidos a partir de pesquisas em bases cientificas, distribuídas a partir das referências por autor, ano, objetivo do trabalho, os materiais utilizados e as suas respectivas considerações finais.

\begin{tabular}{|c|c|c|c|c|}
\hline Autores & Ano & Objetivo & Materiais & Considerações finais \\
\hline ALKHUDHAIRY FI & 2017 & $\begin{array}{l}\text { Investigar os efeitos de duas } \\
\text { intensidades de luz de cura nas } \\
\text { propriedades mecânicas de } \\
\text { compósitos à base de resina de } \\
\text { enchimento a granel (RBCs). }\end{array}$ & $\begin{array}{c}\text { (Tetric }{ }^{\circledR} \mathrm{N} \text {-Ceram, SonicFill TM, inteligente de } \\
\text { substituição Dentina (SDR }{ }^{\mathrm{TM}} \text { ) posterior Fluido } \\
\text { de materiais, e Filtek }{ }^{\mathrm{TM}} \text { posterior restauradora) } \\
\text { foram utilizados neste estudo. }\end{array}$ & $\begin{array}{l}\text { SonicFill mostrou a maior microdureza e } \\
\text { resistência à compressão } \\
\text { significativamente tanto para curar } \\
\text { intensidades de luz quanto para maior } \\
\text { resistência à tração diametral com luz } \\
\text { de alta intensidade. }\end{array}$ \\
\hline ALSHALI RZ, et al. & 2015 & $\begin{array}{c}\text { Medir a razão de dureza inferior / } \\
\text { superior de materiais a granel e } \\
\text { compostos de resina convencionais } \\
\text { e avaliar as alterações de dureza } \\
\text { após o armazenamento a seco e } \\
\text { etanol. }\end{array}$ & $\begin{array}{l}\text { SureFil SDR, Venus a granel, base X-tra, } \\
\text { Filtek com preenchimento a granel, } \\
\text { preenchimento Sonic e Tetric EvoCeram e oito } \\
\text { materiais compósitos de resina convencionais } \\
\text { (fluxo Grandioso, fluxo Venus Diamond, fluxo } \\
\text { X, Filtek Supreme Ultra Flowable, Grandioso, } \\
\text { Venus Diamond, TPH Spectrum e Filtek Z250) } \\
\text { foram testados }(n=5) \text {. }\end{array}$ & $\begin{array}{c}\text { A profundidade de cura recomendada } \\
\text { pelo fabricante para os compósitos de } \\
\text { resina de enchimento a granel pode ser } \\
\text { alcançada com base no método de } \\
\text { microdureza. }\end{array}$ \\
\hline $\begin{array}{c}\text { GONÇALVES F, et } \\
\text { al. }\end{array}$ & 2018 & $\begin{array}{c}\text { Avaliar o grau de conversão, } \\
\text { encolhimento volumétrico pósgel e a } \\
\text { citotoxicidade de seis compósitos } \\
\text { granulométricos e dois compósitos } \\
\text { convencionais }\end{array}$ & $\begin{array}{c}\text { Filtek Z350 XT (Z350) / 3M ESPE; Filtek Z350 } \\
\text { XT fluida (ZF) / 3M ESPE; Aura Bulk Fill (AB) / } \\
\text { SDI; everX Posterior (EP) / GC Europa; } \\
\text { SonicFill (SF) / Kerr; Filtek Bulk Fill Posterior } \\
\text { (FBP) / 3M ESPE; Fluxo de enchimento a } \\
\text { granel Filtek (FBF) / 3M ESPE; Fluxo de } \\
\text { enchimento a granel (VB) da Venus / Heraeus } \\
\text { Kulzer. }\end{array}$ & $\begin{array}{l}\text { As resinas Bulk Fill apresentam elevado } \\
\text { Grau de Conversão (GC), superior à } \\
\text { resina convencional estudada; a nova } \\
\text { classe de materiais restauradores é } \\
\text { capaz de polimerizar em profundidade e } \\
\text { alguns materiais restauradores é capaz } \\
\text { de polimerizar em profundidade. }\end{array}$ \\
\hline
\end{tabular}

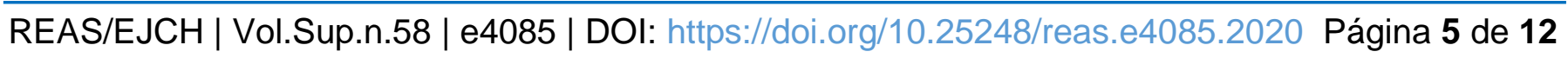




\begin{tabular}{|c|c|c|c|c|}
\hline ALSHALI R Z, et al. & 2013 & $\begin{array}{l}\text { Avaliar o grau de conversão (DC) ao } \\
\text { longo do tempo, utilizando } \\
\text { espectroscopia FTIR para materiais } \\
\text { compósitos de resina fluidos de } \\
\text { enchimento a granel, em } \\
\text { comparação com materiais } \\
\text { compósitos de resina fluidos } \\
\text { convencionais e regulares. }\end{array}$ & $\begin{array}{l}\text { SureFil SDR (SDR), Venus a granel (VBF), x- } \\
\text { tra base (XB) e Filtek Bulk Fill (FBF). Os } \\
\text { materiais compostos fluidos e regulares } \\
\text { convencionais incluem: fluxo Venus Diamond } \\
\text { (VDF), fluxo Grandioso (GRF), Venus } \\
\text { Diamond (VD) e Grandioso (GR). }\end{array}$ & $\begin{array}{c}\text { Os valores de CD pós-cura de } 24 \text { horas } \\
\text { dos compósitos de enchimento a granel } \\
\text { SDR e VBF são geralmente } \\
\text { comparáveis aos dos compósitos } \\
\text { convencionais estudados; no entanto, } \\
\text { os valores de DC de } 24 \text { horas após a } \\
\text { cura de XB e FBF foram menores em } \\
\text { comparação com os outros materiais. }\end{array}$ \\
\hline TAUBOCK T, et al. & 2018 & $\begin{array}{l}\text { Investigar o encolhimento da } \\
\text { polimerização, o desenvolvimento da } \\
\text { força de contração e o grau de } \\
\text { conversãa do monômero em } \\
\text { compósitos bulk fill com base em } \\
\text { dimetacrilato de alta e baixa } \\
\text { viscosidade. }\end{array}$ & $\begin{array}{l}\text { Dois compósitos bulk fill (SDR, base x-tra), } \\
\text { dois compósitos de alta viscosidade (Bulk } \\
\text { Ormocer, SonicFill) e dois materiais } \\
\text { compósitos convencionais (Esthet X flow, } \\
\text { Esthet X HD) foram fotoativados por } 20 \mathrm{~s} \mathrm{em} \\
1275 \mathrm{~mW} / \mathrm{cm} 2 \text {. }\end{array}$ & $\begin{array}{c}\text { No geral, compósitos de preenchimento } \\
\text { a granel com alta quantidade de carga e } \\
\text { baixa taxa de força apresentaram as } \\
\text { características mais favoráveis da força } \\
\text { de retração que suporta seu uso na } \\
\text { restauração de cavidades posteriores } \\
\text { com alto fator C. }\end{array}$ \\
\hline $\begin{array}{c}\text { EL-DAMANHOURY } \\
\text { H e PLATT J }\end{array}$ & 2014 & $\begin{array}{c}\text { Avaliar a cinética de tensão de } \\
\text { contração por polimerização de cinco } \\
\text { compósitos de resina de enchimento } \\
\text { a granel com baixo encolhimento e } \\
\text { baixa contração. }\end{array}$ & $\begin{array}{c}\text { Filtek Z250 (FZ, 3M ESPE) foi usado como } \\
\text { controle. A tensão de retração em tempo real } \\
\text { dos compósitos investigados foi medida } \\
\text { usando um tensômetro; tensão máxima de } \\
\text { contração, taxa de tensão (Rmax) e tempo } \\
\text { para atingir a taxa de tensão máxima (tmax) } \\
\text { foram registrados. }\end{array}$ & $\begin{array}{c}\text { Todos os materiais de preenchimento } \\
\text { em massa testados foram capazes de } \\
\text { alcançar uma eficiência de cura } \\
\text { aceitáael ( } \geq 80 \% \text { percentual inferior / } \\
\text { superior) a } 4 \mathrm{~mm} \text { de profundidade. Em } \\
\text { conclusão, este estudo relata uma } \\
\text { reduçãa significativa no estresse de } \\
\text { contração da polimerização, mantendo } \\
\text { uma eficiência de cura comparável em } 4 \\
\text { mm para alguns compósitos de } \\
\text { preenchimento a granel e suporta seu } \\
\text { uso potencial em situações clínicas } \\
\text { posteriores. }\end{array}$ \\
\hline
\end{tabular}

REAS/EJCH | Vol.Sup.n.58 | e4085 | DOI: https://doi.org/10.25248/reas.e4085.2020 Página 6 de 12 


\section{Revista Eletrônica Acervo Saúde / Electronic Journal Collection Health ｜ ISSN 2178-2091}

\begin{tabular}{|c|c|c|c|c|}
\hline KAYA MS, et al. & 2018 & $\begin{array}{c}\text { Comparar o grau de polimerização } \\
\text { da resina do tipo bulk-fill com três } \\
\text { diferentes unidades de } \\
\text { fotopolimerização. }\end{array}$ & $\begin{array}{l}\text { O composto utilizado foi Giomer (Beautifil-Bulk } \\
\text { Restorative), a partir de três unidades } \\
\text { fotopolimerizáveis: polywave (Valo); } \\
\text { monowave (DemiUltra: DU); e um LED de } \\
\text { segunda geração (Optima 10: Opt) }\end{array}$ & $\begin{array}{l}\text { Os valores de CS foram semelhantes, } \\
\text { mas o módulo de compressão e a } \\
\text { tensão de fraturamento (\%) variaram, } \\
\text { dependendo do protocolo de cura. Com } \\
\text { base nos resultados, pode-se concluir } \\
\text { que o dispositivo e o protocolo de cura } \\
\text { afetam fortemente as reações de } \\
\text { reticulação e, portanto, os valores de\% } \\
\text { DC, SMH, módulo de compressão e } \\
\text { tensão na ruptura. Consequentemente. }\end{array}$ \\
\hline $\begin{array}{l}\text { CANEPPELE T e } \\
\text { BRESCIANI E }\end{array}$ & 2016 & $\begin{array}{c}\text { Descrever as características dos } \\
\text { materiais disponíveis no mercado, } \\
\text { assim como os resultados clínicos já } \\
\text { descritos na literatura, na tentativa } \\
\text { de informar e dar suporte para a } \\
\text { decisão de emprego ou não deste } \\
\text { tipo de materiais. }\end{array}$ & $\begin{array}{l}\text { Artigos científicos dos anos de } 2014,2015 \text { e } \\
2016 \text {, disponíveis no Pubmed, contendo } \\
\text { trabalhos que comparassem as resinas bulk } \\
\text { com resinas convencionais, para que o } \\
\text { panorama atual pudesse ser traçado. }\end{array}$ & $\begin{array}{c}\text { Este novo grupo de materiais } \\
\text { representa uma possibilidade para } \\
\text { restaurações diretas, principalmente } \\
\text { pela facilidade de técnica e similaridade } \\
\text { de propriedades em comparação com } \\
\text { as resinas convencionais. Não se sabe } \\
\text { frente ao exposto, se estes materiais } \\
\text { devem ou podem substituir as resinas } \\
\text { convencionais, sendo que mais estudos } \\
\text { e controles clínicos maiores são } \\
\text { necessários. }\end{array}$ \\
\hline $\begin{array}{l}\text { FERREIRA AB e } \\
\text { SILVA NEF }\end{array}$ & 2017 & $\begin{array}{l}\text { Mostrar as vantagens do uso clínico } \\
\text { das resinas Bulk Fill em relação às } \\
\text { resinas compostas convencionais. }\end{array}$ & $\begin{array}{l}\text { Foi realizado um levantamento bibliográfico, } \\
\text { com a utilização de artigos científicos, } \\
\text { monografias e publicações que possuem } \\
\text { conteúdos relevantes ao texto deste artigo. } \\
\text { Para isso, foram acessados artigos em } \\
\text { português e inglês, entre } 2006 \text { e } 2017 \text {, nas } \\
\text { bases de dados PubMed e Bireme. }\end{array}$ & $\begin{array}{c}\text { As RCBF estão e podem ser indicadas } \\
\text { para a restauração de dentes } \\
\text { posteriores. Entretanto, dureza e } \\
\text { contração de polimerização têm relação } \\
\text { direta com o tempo e potência de } \\
\text { irradiação do aparelho fotoativador. }\end{array}$ \\
\hline
\end{tabular}

Fonte: Neto JMAS, et al, 2020.

REAS/EJCH | Vol.Sup.n.58 | e4085 | DOI: https://doi.org/10.25248/reas.e4085.2020 Página 7 de 12 


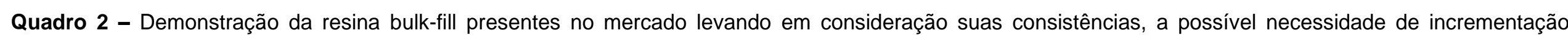
superficial com resina convencional e seu mecanismo de ativação.

\begin{tabular}{|c|c|c|c|c|c|}
\hline Fabricante & $\begin{array}{l}\text { Relação de } \\
\text { consistência }\end{array}$ & $\begin{array}{l}\text { Incrementação } \\
\text { máxima }\end{array}$ & $\begin{array}{l}\text { Necessidade de } \\
\text { incrementação } \\
\text { com resina } \\
\text { convencional }\end{array}$ & $\begin{array}{c}\text { Mecanismo responsável pela contração } \\
\text { reduzida }\end{array}$ & Cores disponíveis \\
\hline $\begin{array}{l}\text { Surefil SDR } \\
\text { flow } \\
\text { (Dentsply) }\end{array}$ & Fluida & $4 \mathrm{~mm}$ & Sim & $\begin{array}{c}\text { Presente na estrutura do monômero resinoso } \\
\text { moduladores - apresentam menores ligações } \\
\text { cruzadas e regulamentação das cadeias } \\
\text { poliméricas. }\end{array}$ & U (universal), A1, A2 e A3 \\
\hline $\begin{array}{l}\text { Filtek Bulk Fill } \\
\text { Flow (3M) }\end{array}$ & Fluida & $4 \mathrm{~mm}$ & Sim & $\begin{array}{c}\text { Alto peso molecular e alta fluidez: BisGMA, } \\
\text { BisEMA, Procrylat e UDMA. }\end{array}$ & $\mathrm{A} 1, \mathrm{~A} 2, \mathrm{~A} 3 \mathrm{e} \mathrm{U}$ \\
\hline $\begin{array}{l}\text { x-tra base } \\
\text { (VOCO) }\end{array}$ & Fluida & $4 \mathrm{~mm}$ & Sim & $\begin{array}{l}\text { Sua carga presente corresponde em } 75 \% \text { de } \\
\text { monômeros especiais. }\end{array}$ & U ou A2 \\
\hline $\begin{array}{l}\text { Opus Bulk Fill } \\
\text { flow (FGM) }\end{array}$ & Fluida & $4 \mathrm{~mm}$ & Sim & Balanceamento entre a carga e os monômeros. & $\mathrm{A} 1, \mathrm{~A} 2$ e $\mathrm{A} 3$ \\
\hline $\begin{array}{l}\text { Filtek Bulk Fill } \\
\qquad(3 \mathrm{M})\end{array}$ & Regular & $5 \mathrm{~mm}$ & Não & $\begin{array}{l}2 \text { monômeros: AUDMA - reduzindo assim } \\
\text { ligações cruzadas e AFM - cliva a cadeia } \\
\text { durante o processo de polimerização. }\end{array}$ & $\mathrm{A} 1, \mathrm{~A} 2, \mathrm{~A} 3$ e $\mathrm{B} 1$ \\
\hline $\begin{array}{l}\text { comercial6. } \\
\text { apcd@ } \\
\text { apcdcentral. } \\
\text { com.br }\end{array}$ & Regular & $4 \mathrm{~mm}$ & Não & $\begin{array}{c}\text { Carga correspondente a } 85 \% \text { de monômeros } \\
\text { especiais. }\end{array}$ & U (universal) \\
\hline $\begin{array}{l}\text { Admira } \\
\text { Fusion x-tra } \\
\text { (VOCO) }\end{array}$ & Regular & $4 \mathrm{~mm}$ & Não & Apresenta moléculas de ligação ORMOCER. & U (universal) \\
\hline $\begin{array}{l}\text { Tetric N- } \\
\text { Ceram Bulk } \\
\text { Fill (Ivoclar } \\
\text { Vivadent) }\end{array}$ & Regular & $4 \mathrm{~mm}$ & Não & $\begin{array}{l}\text { Fotoiniciador correspondente a base de } \\
\text { germânio. }\end{array}$ & $\begin{array}{c}\text { IVA, IVB e IVW (para dentes A, B e } \\
\text { clareados) }\end{array}$ \\
\hline $\begin{array}{l}\text { SonicFill } \\
\text { (Kerr) }\end{array}$ & Regular & $4 \mathrm{~mm}$ & Não & Energia sônica - diminuição da viscosidade. & $A 1, A 2, A 3$ ou $B 1$ \\
\hline $\begin{array}{l}\text { Aura Bulk Fill } \\
\text { (SDI) }\end{array}$ & Regular & $4 \mathrm{~mm}$ & Não & Não informado. & U (universal) \\
\hline
\end{tabular}

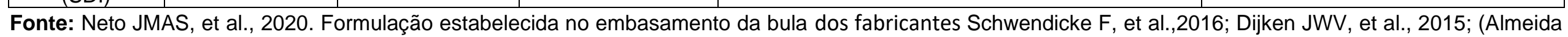
LJDS, et al., 2017. 
A utilização das resinas compostas $(\mathrm{RC})$ tem se fortalecido nas últimas décadas, de modo especial por causa das suas particularidades presentes, dos quais favoreceu sua estética. Contudo, além de que, os princípios encontrados nos compostos resinosos apresentam diferentes atributos que fundamentam sua aplicação em grandes dimensões, a começar sobre sua resistência, seu custo alcançável, compatibilidade e a probabilidade de aprestos cavitários anatômicos bem mais favorável em sua conservação e implemento. (KAYA MS, et al., 2018).

Contudo, as resinas compostas da mesma forma que outros agentes utilizados em restaurações possuem as vezes determinados entraves que não ultrapassam os seus benefícios, uns dos entraves seriam relacionados as características mecânicas presentes, contração de polimerização desde os fotopolimerizáveis aos autopolimerizáveis, processos relacionados a possíveis agentes tóxicos e inconstância ou até mesmo sua mudança de cor ao longo do tempo (KAYA MS, et al., 2018). Essas restrições são capazes de baixar enormemente a durabilidade de conservação das restaurações. No que concerne a conquista de um agradável padrão estético e duradouro, a modificação da tonalidade aplicada nas restaurações com compostos se estabelece como sendo um incitamento para os profissionais da área e os cientistas. Esses impasses estão correlacionados a inúmeros fatores, estando como formas de exemplificações os aspectos intrínsecos e extrínsecos. As condições intrínsecas são relativas com sua forma de firmeza química presente na matriz resinosa, enquanto os aspectos extrínsecos, estão agregados às situações de potencialidade de agressão contínuo e circunstâncias desiquilibrado do meio oral. Tais situações diversificam desde a presença de corantes presentes na dieta do paciente (FESTUCCIA MS, et al., 2012).

\section{Classificação}

As resinas do tipo bulk fill, são comercializadas através de duas apresentações distintas: uma dela é mais fluida, pode-se preencher em apenas um incremento de $4 \mathrm{~mm}$ de espessura, e logo após a sua polimerização é finalizada a restauração com uma camada de $2 \mathrm{~mm}$ de resina hibrida ou nanoparticulada, essas resinas com baixa quantidade de carga, ou seja, com menor viscosidade são consideradas fluidas, dos quais possuem entre 20 a $25 \%$ a menos de percentual de carga, devido esse processo viscoso ser reduzido essas resinas são utilizadas como base por causa do seu baixo modulo de elasticidade (KAYA MS, et al., 2018).

As resinas bulk-fill que apresentam alta quantidade de carga, ou seja, grande viscosidade são consideradas regulares, essa maior quantidade de carga faz com quer os compostos consigam suportar o estresse funcional e mecânico, quando há uma diminuição da sua energia funcional, ou seja de uma energia sônica para uma melhor adaptação, com isso o cirurgião dentista é capaz de preencher cavidades de até $5 \mathrm{~mm}$ de profundidade e em seguida esculpir e fotopolimerizar o local estabelecido do tratamento (TAUBOCK T, et al., 2018). Estudos relatam que essa nova categoria de resina apresenta uma atividade de cura de 4 a $5 \mathrm{~mm}$, dependendo da intensidade gerada do fotopolimerizador irá ser preciso maior tempo de fotopolimerização para obter a mesma energia, a energia necessária é a mesma de uma resina convencional, isso ocorre porque uma polimerização a uma determinada profundidade não depende somente da quantidade de fótons chegando até ela, depende também da polimerização iniciada nas camadas superiores, dos quais é conhecido como propagação em profundidade (KAYA MS, et al., 2018).

\section{Vantagens}

A redução de contração de polimerização trouxe benefícios ao que se refere a essa classe de compostos restauradores, pois a tensão de contração quando presente pode gerar deformações das cúspides, sensibilidades, trincas em esmalte ou dentina, falhas adesivas na interface dente/restauração, processos de cáries secundárias e infiltrações. Uma maior fluidez dos compostos permite melhor adaptação a cavidade que está sendo preparada, comparado com as resinas convencionais sua restauração é rápida, devido ser uma técnica de preenchimento único em que ocorre uma simplificação técnica, pois existe uma redução no protocolo convencional e com isso demanda-se um menor tempo clinico. Além dessas vantagens uma que se destaca é a menor formação de bolhas, isso ocorre porque o incremento inserido na cavidade existe menor retenção de espaços vazios (CHESTERMAN J, et al., 2017). Estudos feitos por ALKHUDHAIRY FI (2017); relatam que devido a fluidez desses compostos eles se adaptam melhor a cavidade, dificultando assim 0 aprisionamento de ar e consequentemente redução significativa de bolhas. 


\section{Desvantagens}

Ocorre falha de restauração quando o incremento passa de $5 \mathrm{~mm}$ na cavidade, ocorre rugosidade da superfície quando exposta a bebidas ácidas e alcoólicas e não apresentam adaptações marginais superiores que as resinas compostas convencionais (CHESTERMAN J, et al., 2017).

\section{Indicações}

Os compostos da RCBF são constituídos por novos tipos de monômeros químicos, tendo em seus princípios ativos partículas inorgânicas dos quais possui aperfeiçoamento na translucidez e, que por consequência, obtém potencial de atingir um bom nível de transformação, até mesmo nas paredes de fundo da cavidade, da qual a luz do fotopolimerizador é mais difícil de ser alcançada (MONTERUBBIANESI R, et al., 2016).

As RCBF apresentam resultados satisfatórios em relação a restauração de elementos dentais tratados endodonticamente, porém a restauração deve ser feita por um profissional qualificado pois o processo restaurador da parte coronal é o último passo do tratamento proposto, dos quais não observa apenas a restauração em si, mas também propõe o fortalecimento do elemento dental requerido. Quando encontrasse cáries ou trincas na parte coronária do dente, e o processo terapêutico endodôntico é preciso ser realizado, as disposições restantes do dente é ainda mais fragilizada por causa do preparo da cavidade estabelecida na hora do acesso dos canais. Devido esse fator é necessário que o profissional tenha cuidado na hora da incrementação com RCBF para só assim evitar um resultado não satisfatório. (KEMALOGLU H, et al., 2015).

Foram realizados estudos onde o principal objetivo era analisar a fixação ou as possíveis modificações de tonalidade da coloração presentes nas resinas compostas do tipo Bulk Fill, para pode ser feito as observações e analises foram emergidas as resinas em princípios ativos que apresentavam corantes, esse estudo se direcionou por falta de pesquisas focada nesse contexto. Similar aos estudos que empregavam em seus experimentos compostos convencionais deixando-os submersos a líquidos corantes, frequentemente vigentes na dieta dos pacientes, as substâncias utilizadas como fonte de analise foram: A água destilada como forma de comparação e controle, e o vinho e café como resultados, o resultado final foi que as resinas que ficaram submersas ao vinho e o café tiveram uma variação de coloração (ELSHARKASI M, et al., 2015); (DIJKEN JWV, et al., 2015).

Os alimentos que ocasionam pigmentações presentes nas dietas dos pacientes são na maioria das vezes os de formas fluidas, os mesmos vem sendo instrumentos de atuais analises de pesquisas, cujo estudo tencionam fazer comparação dos eventuais líquidos ao que se expõe as pigmentações dos elementos dentais, e comensurar a aptidão de modificação das cores nas restaurações estabelecidas (ALMEIDA LJDS, et al., 2017). As mudanças da tonalidade das restaurações feitas com essas resinas especificas podem acontecer devido dois fatores importantes: A adsorção ou absorção provindas de fontes externas, como foi mencionado anteriormente o café, corantes, além de chá, pacientes tabagistas e determinados tipos de enxaguantes orais (ELSHARKASI M, et al., 2015).

De acordo com Nahsan FPS, et al. (2009), onde teve seus estudos relacionados a análise das estabilidades das cores feitas pelas restaurações com resinas do tipo bulk fill através do equipamento que mede radiações eletromagnéticas, foi constatado exemplos em duas substâncias, café e clorexidina a 0,12\%, sendo a água destilada o grupo controle, ao fazer o experimento ficou notório que o grupo que estava com café apresentou maior prevalência de pigmentações e alterações da coloração da resina.

Apesar de existirem vários experimentos através de estudos das consequências de distintas dietas relacionadas a bebidas na estabilidade da coloração da $\mathrm{RC}$, existem poucas informações ao que se refere a estabilidade de cor nas resinas do tipo bulk-fill, visto que essa resina é utilizada em camadas mais densas (ELSHARKASI M, et al., 2015). Shamszadeh S, et al. (2016) analisaram a estabilidade do processo de tonalidade de cor das resinas bulk-fill e convencional com dessemelhantes espessuras quando submergidas em café e logo em seguida em água. 
Os pesquisadores evidenciaram em seu trabalho que a RCBF denotou elevada vulnerabilidade à tonalidade da coloração logo após a submersão no recipiente que possuía café quando contraposto a RC. Devido ao fato da incrementação ser mais consistente, conseguiu deduzir que o processo de descoloração se eleva de acordo com a densidade do incremento. Relataram da mesma forma que a maior vulnerabilidade à pigmentação da coloração dos incrementos na resina bulk-fill decorrem por causa da sua menor profundidade ao que se refere à forma de sua polimerização.

Um alto grau de modificação pode estabelecer satisfatórias respostas ao que se refere ao processo mecânico, equilíbrio químico, além da durabilidade da restauração executada. Na realidade, o valor da modificação é bastante crucial para analisar de forma mais significativa as propriedades físicas, mecânicas e biológicas dos compósitos das resinas do tipo Bulk Fill, de outra forma, quando não se estabelece um bom padrão de conversão, podem acontecer uma forma de fotopolimerização incompleta, decorrendo assim infiltrações marginais, modificação da tonalidade da resina, além de diminuir significativamente da adesão e baixar as propriedades mecânicas estabelecidas (MONTERUBBIANESI R, et al., 2016).

Dijken JWV, et al. (2015) acompanharam a estabilidade clínica perdurante entre 3 anos das resinas bulkfill em tratamentos restauradores referente as Classes I e II. Sendo a classe I Cavidades criada em locais de má coalescência dos esmaltes, locais de cicatrículas e fissuras da área oclusal dos elementos dentais molares e pré-molar, $2 / 3$ oclusal da face vestibular dos molares inferiores, lingual dos incisivos e caninos superiores, 2/3 a face palatina dos molares superiores, já a classe II são cavidades criadas nas partes presentes nas áreas proximais dos pré-molares e molares do qual não foi observada nos pacientes nenhuma forma de sensibilidade pós a realização da restauração.

Nos 3 anos, foram observadas e analisadas 196 restaurações, das quais 74 foram realizadas com Classe I e 122 com restaurações de Classe II, pode ser visto que sete restaurações apresentaram falhas (3,6\%), 4 restaurações sendo de resina nanohíbrida e 3 restaurações- CeramX mono + , todas as falhas sendo de Classe II.

Um dos principais fatos dessas intercorrências foi devido a fraturas ocorridas nos dentes, enquanto as outras ocorreu devido a fratura da resina. Essas falhas ocorridas nesse período ao que se refere as falhas na Classe I e II, representou uma porcentagem de 1,2\% nas RCBF enquanto a RC representou $1 \%$. A técnica da resina bulk-fill demostrou segmentos clínicos positivos e sendo bastante parecido com a técnica incremental no decorrer dos 3 anos. Sendo observado ótimas qualidades na parte superficial, adequação da parte marginal e grande estabilidade da coloração, bem como observado baixa frequência de caries secundárias, quando realizada com boa técnica.

\section{CONSIDERAÇÕES FINAIS}

De acordo com a resolução da literatura associada, foi possível percebe-se que, na maior parte dos casos, as resinas do tipo bulk fill apontam propriedades parecidas com as resinas convencionais, no momento que é seguido o procedimento padrão ao que se refere à cada uma delas. A utilização das RCBF facilita o cotidiano do Cirurgião Dentista por diminuir de forma significativa vários passos e etapas que as resinas convencionais necessitam, as resinas compostas do tipo Bulk fill apontam certa estabilidade de cor quando se comparada com as resinas compostas tradicionais, levando em consideração que as RCBF obtiveram grau de manchamento extrínseco parecidos, porém as resinas Bulk fill demostrou maior tonalidade quando se submeteu ao vinho enquanto as resinas tradicionais ao café, isso se deve ao fato de que as Bulk fill apresentam maior força ao que se refere a compressão, possuindo elevada habilidade de suportar tensão e deformação do que as RC. As RCBF retêm uma conexão bastante direta com a dimensão da radiação do aparelho fotoativador cooperando de forma direta para o melhor funcionamento, tonalidade e velocidade no trabalho dos Cirurgiões Dentistas. Até o pressente momento, estudos clínicos longitudinais são fundamentais serem feitos para o estabelecimento preciso do desempenho das Resinas Compostas do tipo bulk fill. 


\section{REFERÊNCIAS}

1. ALKHUDHAIRY FI. The effect of curing intensity on mechanical properties of different bulk-fill composite resins. Clin Cosmet Investig Dent, Kingdom of Saudi Arabia, 2017; 1-6.

2. ALMEIDA LJDS, et al. Is there correlation between polymerization shrinkage, gap formation, and void in bulk fill composites? A $\mu$ CT study. Brazilian Oral Research, 31, 2017; 1-10.

3. ALSHALI RZ, et al. Degree of conversion of bulk-fill compared to conventional resin-composites at two time intervals. Dental Materials, 2013; 29(9); 213-217.

4. ALSHALI RZ, et al. Post-irradiation hardness development, chemical softening, and thermal stability of bulk-fill and conventional resin-composites. J Dent, Manchester, 2015; 43(2): 209-218.

5. ANUSAVICE KJ. Phillips: materiais dentários. 11.ed. Rio de Janeiro: Elsevier, 2005; 800.

6. CANEPPELE T, BRESCIANI E. Resinas bulk-fill - O estado da arte. 2016. Revisão de literatura. Departamento de Odontologia Restauradora do Instituto de Ciência e Tecnologia do Campus de São José dos Campos - Unesp, 2016

7. CHESTERMAN J, et al. Bulk-fill resin-based composite restorative materials: a review. British Dental Journal, Manchester, 2017; 222(5): 337-344.

8. DIJKEN JWV, et al. Randomized 3-year clinical evaluation of class I and II posterior resin restorations placed with a bulk fill resin composite and a one-step self-etching adhesive. J Adhes Dent, New Malden, 2015; 17(1): 81-88.

9. EL-DAMANHOURY H, PLATT J. Polymerization shrinkage stress kinetics and related properties of bulk-fill resin composites. Oper Dent, 2014; 39(4): 374-82.

10. ELSHARKASI M, et al. Cuspal deflection in premolar teeth restored with bulk-fill resinbased composite materials. Tese (Master of Science in Dentistry) - Indiana University School of Dentistry, Indiana, 2015.

11. FERRACANE JL. Resin composite--state of the art. Dent Mater, Portland, 2011; 27(1): 29-38.

12. FERREIRA AB, SILVA NEF. Utilização das Resinas Compostas Bulk Fill: uma revisão da literatura. 2017. Trabalho de conclusão de curso (Bacharel em Odontologia) - Faculdade Integrada de Pernambuco, Pernambuco, 2017.

13. FESTUCCIA MS, et al. Color stability, surface roughness and microhardness of composites submitted to mouthrinsing action. J Appl Oral Sci. 2012; 20(2): 200.

14. FLURY S, et al. Influence of increment thickness on microhardness and dentin bond strength of bulk fill resin composites. Dent Mater, Bern, 2014; 30(10): 1104-1112.

15. GONÇALVES F, et al. A comparative study of bulk-fill composites: degree of conversion, post-gel shrinkage and cytotoxicity. Braz Oral Res, São Paulo, 2018; 32.

16. KAYA MS, et al. Structural and mechanical properties of a giomer-based bulk fill restorative in different curing conditions. J Appl Oral Sci, Turkey, 2018.

17. KEMALOGLU H, et al. Effect of novel restoration techniques on the fracture resistance of teeth treated endodontically: an in vitro study. Dental Material Journal, Washington, 2015; 34(5): 618-622.

18. MONTERUBBIANESI R, et al. Spectroscopic and mechanical properties of a new generation of bulk fill composites. Frontiers in Physiology, Ohio, v. 7, no. 652, 2016; p. 1-9.

19. NAHSAN FPS, et al. Estabilidade de cor de resina composta após imersão em café, água e solução de clorexidina. Revista Brasileira de Pesquisa em Saúde/Brazilian Journal of Health Research, 2009.

20. RODRIGUES J, COSTA E. Estudo de propriedades de resinas compostas bulk fill. 2015. Dissertação de Mestrado (Odontologia) - USP, São Paulo, 2016.

21. SHAMSZADEH S, et al. Color stability of the bulk fill composite resins with different thickness in response to coffe/ water immersion. Int J Dent. 2016; p. 1-5.

22. SILVA NETO JM, et al. O uso das resinas compostas tipo bulk fill: Uma revisão de literatura. Revista Eletrônica Acervo Saúde, (37), e1887, 2019.

23. TAUBOCK T, et al. Polymerization shrinkage and shrinkage force kinetics of high- and low-viscosity dimethacrylateand ormocer-based bulk-fill resin composites, Odontology, Zurich, 2018. 